\title{
A FACULA MODEL AND ITS APPLICATION TO FACULA FINE STRUCTURES
}

\author{
E. WIEHR and G. STELLMACHER \\ Universitäts-Sternwarte, Göttingen, F.R.G.
}

\begin{abstract}
In a recent paper Stellmacher and Wiehr (1973) discussed in detail the discrepancies between 'continuum-' and 'line-profile facula models'. Their calculations show that continuum models (e.g. Kuz'minykh, 1962; Schmahl, 1967; Chapman 1970; Wilson, 1971) encounter the problem that the facula contrast increases towards the limb but decreases with wavelength $\lambda$. This discrepancy evidently cannot be due to NLTE effects since it concerns the continumm data.

Problems increase essentially when line profile data are taken into account. Measurements of several magnetically insensitive lines (Stellmacher and Wiehr, 1971) show that the normalized profiles are identical for facula and neighboring photosphere except for the line cores (rest-intensity effect). These results indicate a temperature excess in high facula layers $\left(\tau_{0} \leqq 0.03 \hat{=} h \geqq 250 \mathrm{~km}\right)$ increasing with height and a photospheric temperature stratification in the deeper facula layers. Such a (preliminary) model (A) also fits to the observed dependence of the rest-intensity effect on the line excitation potential.
\end{abstract}

The continuum contrast at the disc center including its $\lambda$-dependence $(\approx 2 \%$ constant for $4000 \AA \leqq \lambda \leqq 8000 \AA$ ) requires in addition to the temperature stratification of model A, a reduction of the gas pressure by $\Delta \log P_{\mathrm{g}}=-0.015$. The resulting model (C) is then able to represent all facula data observed at the disc center including the magnetic field (when taking $H^{2}=8 \pi \Delta P_{\mathrm{g}}$ ); however, it fails to represent the observed center-to-limb variation of the contrast (CLV). On the other hand, facula models which do fit the CLV strongly contradict the observed $\lambda$-independent contrast and the unchanged line wings.

Hence, we suggest assuming our 'disc center model' $\mathrm{C}$ and trying to explain the CLV exclusively by the facula geometry. Here we draw two possibilities:

(1) Elevation of the geometric facula scale by $200 \mathrm{~km}$ and a finite facula diameter of $2000 \mathrm{~km}$ easily yields the observed CLV. However, whereas the finite facula diameter seems reasonable, the elevation by $200 \mathrm{~km}$ contradicts our $\Delta \log P_{\mathrm{g}}=-0.015$ which actually corresponds to a depression of $10 \mathrm{~km}$.

(2) Applying the considerations on the Wilson effect in sunspots by Jensen et al. (1969) to the facula model and assuming a certain 'curvature' of the depression, one can obtain a CLV of the contrast depending on the assumed curvature.

None of these geometric models would limit the validity of our model C. In particular the data expected for facula fine structures remain compatible with our model: The continuum contrasts of single facula granules should reasonably be larger than those of the smeared-out facula region, but these larger contrasts would still be $\lambda$ - 
independendent (because the smear-out by blurring is $\lambda$-independent). Furthermore, the normalized line profiles of a single facula granule can be expected to remain unchanged in the wings (otherwise our profile observations for smeared-out faculae would not contain one facula granule at all!). Model $\mathrm{C}$ can thus easily be adapted to single facula granules by applying an additional reduction to the gas pressure.

As an example we assumed a 'corrected' contrast of $6 \%$ for a single facula granule. Calculations with our model $\mathrm{C}$ yield this contrast including the required $\lambda$-independence and the unchanged line wings when applying $\Delta \log P_{\mathrm{g}}=-0.06$. Since hydrostatic equilibrium requires a magnetic field $H^{2} \sim P_{\mathrm{g}} \Delta \log P_{\mathrm{g}}$ the increase of $\Delta \log P_{\mathrm{g}}$ by a factor of 4 yields an increase of the magnetic field by a factor of 2 as compared to the values for the smeared-out facula region deduced by Stellmacher and Wiehr (1973). It seems reasonable that the observed contrast and magnetic field for smeared-out facula regions are lower than the actual values for single facula granules. The resulting relation between contrast and magnetic field roughly agrees with the measurements by Frazier (1971).

Our model predictions of a temperature excess exclusively confined to the higher layers $(h \geqq 250 \mathrm{~km})$ together with a pressure reduction relative to the adjacent photosphere fits well to the theoretical 'shock flow model' by Meyer and Schmidt (1968). This model proposes a temperature excess above the $400-\mathrm{km}$ level increasing with height as well as a downward flow with subsonic velocities in the lower facula layers. Such a downward flow, however, usually is observed for facula regions and amounts to about $0.8 \mathrm{~km} \mathrm{~s}^{-1}$ (see e.g. Howard, 1972).

\section{References}

Chapman, G. A.: 1970, Solar Phys. 14, 315.

Frazier, E. N.: 1971, Solar Phys. 21, 42.

Howard, R.: 1972, Solar Phys. 24, 123.

Kuz'minykh, V. D.: 1965, Soviet Astron. 8, 551.

Jensen, E., Brahde, R., and Ofstad, P.: 1968, Solar Phys. 9, 397.

Meyer, F. and Schmidt, H. U.: 1968, Mitt. Deutsche Astron. Ges. 25, 194.

Schmahl, G.: 1967, Z. Astrophys. 66, 81.

Stellmacher, G. and Wiehr, E.: 1971, Solar Phys. 18, 220.

Stellmacher. G. and Wiehr, E.: 1973, Astron. Astrophys. 29, 13.

Wilson, P. R.: 1971, Solar Phys. 21, 107.

\section{DISCUSSION}

Pecker: How is your model with the reduction of the pressure in the photospheric layers by $\Delta \log P_{8}=0.02$ compatible with an increase of the opacity that you suggest for the explanation of the center- to limb variation of the contrast? I would have placed the $\tau=1$ line deeper in the faculae than in the surrounding photosphere. Now a second question. Your Figure No. 2 shows no variation in contrast with wavelength. That is a very intersting observational result. When you go near the limb what does the curve evolve to? Does it still show no variation with wavelength?

Wiehr: Yes, here we confirmed former measurements by Schmahl. I wonder why these observations are not better known, perhaps because they were published in Zeitschrift für Astrophysik in German. Concerning your first comment we are aware of the problem: If you evaluate the isolines from the pressure reduction $\Delta \log P_{\mathrm{g}}=0.015$, you actually get a depression of about $10 \mathrm{~km}$ in contradiction to the proposed 
elevation of $200 \mathrm{~km}$. This discrepancy possibly disappears if you keep in mind that there is a downward motion observed in all faculae which might alter the pressure scale. But perhaps one should follow our second geometric suggestion.

Pecker: There is another solution which I don't like but which I will say and that is that the balance of pressure might be achieved by increasing the magnetic field above the value that you have computed.

Wiehr: Only a magnetic field of very complicated structure would be able to balance the higher facula pressure required for the elevation.

Wilson: Another discrepancy is the difference in the temperatures obtained from the continuum measurements and the line temperatures. What were the temperature values that you would get from the line data?

Wiehr: For the line weakenings we require a temperature difference of about $100^{\circ}$ at $\tau=10^{-3}$, for the center-to-limb variations you need larger temperatures but these definitely contradict the observed wavelength independence of the contract of the continuum.

Thomas: At which level does the temperature increase occur that you require for the representation of the central line weakenings?

Wiehr: At $\tau_{0} \approx 0.03$ corresponding to $h \approx 250 \mathrm{~km}$ above $\tau_{0}=1.0$. All these calculations, of course, are based on the assumption of LTE!

Wison: You mentioned the contradiction between continuum and line models, namely the temperature excess of $500-1000^{\circ}$ required for Wilson's and for Chapman's models and that of $100-200^{\circ}$ required for the line data. I feel that these contradictions clearly indicate that one-dimensional analysis are quite inadequate and that geometry is an essential ingredient in any analyst. In this respect geometry, i.e. a greatly increased surface area, may give rise to departures from LTE in lines which would certainly be in LTE at these levels of a one-dimensional atmosphere. A larger excess temperature will weaken the lines while departures from LTE will strngthen them. Thus the line data may be consistent with larger excess temperatures.

Wiehr: We agree with you that geometry is an essential ingredient, this clearly comes out from our discussion of the facula atmosphere and is the reason behind our proposal of two possible geometric models! On the other hand your NLTE considerations evidently are unable to remove the discrepancies between the continuum models and the observed $\lambda$-independent contrasts. Furthermore, I feel that the unchanged line wings give very strong indication for photospheric conditions in deeper facula layers $\left(\tau_{0}>0.03\right)$ rather than for a non-photospheric temperature stratification the influence of which on the line profiles being exactly counter-balanced by NLTE. Last, but not least, the line strengthening by NLTE you mentioned would affect the line cores more than the wings, whereas the contrary would be required: The discrepancy between the continuum models and the observed line profiles is very strong in the wings and nearly absent in the cores (see Figure 4 of our paper). 